Article

\title{
Securing IoT-Based RFID Systems: A Robust Authentication Protocol Using Symmetric Cryptography
}

\author{
Khwaja Mansoor ${ }^{1,+}$, Anwar Ghani $2,+\left(\right.$ C) Shehzad Ashraf Chaudhry ${ }^{3,+}+$, \\ Shahaboddin Shamshirband $4,5, *,+\oplus$, Shahbaz Ahmed Khan Ghayyur ${ }^{2,+}$ and Amir Mosavi $6,7,+[$ \\ 1 Department of Computer Science, Air University Islamabad, Islamabad 44000, Pakistan; \\ kh.mansoorulhassan@gmail.com \\ 2 Department of Computer Science \& Software Engineering, International Islamic University Islamabad, \\ Islamabad 44000, Pakistan; anwar.ghani@iiu.edu.pk (A.G.); shahbaz.ahmed@iiu.edu.pk (S.A.K.G.) \\ 3 Department of Computer Engineering, Faculty of Engineering and Architecture, Istanbul Gelisim University, \\ Istanbul 34310, Turkey; Shahzad@iiu.edu.pk \\ 4 Department for Management of Science and Technology Development, Ton Duc Thang University, \\ Ho Chi Minh City, Viet Nam \\ 5 Faculty of Information Technology, Ton Duc Thang University, Ho Chi Minh City, Viet Nam \\ 6 Faculty of Health, Queensland University of Technology, Victoria Park Road, Kelvin Grove, QLD 4059, \\ Australia; amir.mosavi@kvk.uni-obuda.hu \\ 7 Kando Kalman Faculty of Electrical Engineering, Obuda University, 1034 Budapest, Hungary \\ * Correspondence: shahaboddin.shamshirband@tdtu.edu.vn \\ + These authors contributed equally to this work.
}

Received: 22 July 2019; Accepted: 12 September 2019; Published: 1 November 2019

check for updates

\begin{abstract}
Despite the many conveniences of Radio Frequency Identification (RFID) systems, the underlying open architecture for communication between the RFID devices may lead to various security threats. Recently, many solutions were proposed to secure RFID systems and many such systems are based on only lightweight primitives, including symmetric encryption, hash functions, and exclusive OR operation. Many solutions based on only lightweight primitives were proved insecure, whereas, due to resource-constrained nature of RFID devices, the public key-based cryptographic solutions are unenviable for RFID systems. Very recently, Gope and Hwang proposed an authentication protocol for RFID systems based on only lightweight primitives and claimed their protocol can withstand all known attacks. However, as per the analysis in this article, their protocol is infeasible and is vulnerable to collision, denial-of-service (DoS), and stolen verifier attacks. This article then presents an improved realistic and lightweight authentication protocol to ensure protection against known attacks. The security of the proposed protocol is formally analyzed using Burrows Abadi-Needham (BAN) logic and under the attack model of automated security verification tool ProVerif. Moreover, the security features are also well analyzed, although informally. The proposed protocol outperforms the competing protocols in terms of security.
\end{abstract}

Keywords: authentication protocol; IoT Security; RFID security; symmetric cryptography

\section{Introduction}

Since its inception, the Internet of Things (IoT) is an emerging idea and is defined as, "A system of interrelated computing devices, mechanical and digital machines, objects, animals, or people that are provided with unique identifiers (UIDs) and the ability to transfer data over a network without requiring human-to-human or human-to-computer interaction" [1]. The devices are equipped with 
internet and are capable of communicating with other devices, and such systems are administered and monitored remotely [2,3]. The IoT assimilates heterogeneity of networks, such as smart cities, sensor networks, smart grids, Radio Frequency Identification (RFID), and transportation and parking systems. The RFID is also on its way to replace conventional bar code systems, as the latter have limitations, including line of sight communication, very limited storage capacity, and prone to physical damage. The overall RFID system architecture is depicted in Figure 1.

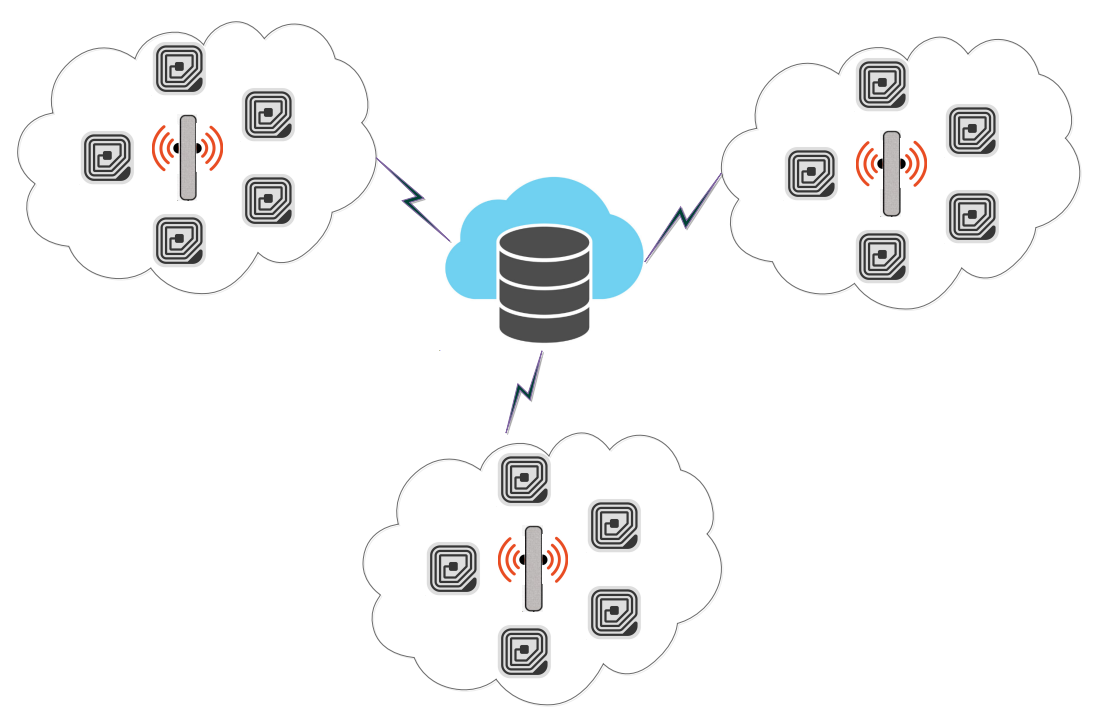

Figure 1. Radio Frequency Identification (RFID) System Architecture.

RFID is simplest form of pervasive sensor networks and is commonly used for identification of physical objects [4,5]. Systems based on RFID consist of a tag, which is equipped with a transceiver to send and receive radio signals from connected devices [6,7]. The RFID Reader is the other device which acts as an access point and can receive and send messages to transceivers. The Reader is also responsible for the availability of tag information at application level [8-10]. RFID tags can be passive, as well as active. Table 1 summarizes the features of passive and active tags.

Table 1. RFID-tag features.

\begin{tabular}{lll}
\hline Features & Passive Tags & Active Tags \\
\hline Data Storage & 128 bytes & 128 bytes \\
tag Power & Energy transferred through Radio Frequency from Reader & Internal source to tag \\
tag Battery & No & Yes \\
Availability of Source Power & Only in range of Radar & Continuous \\
Signal Strength required to tag & Very High & Very Low \\
Range & Upto 3-5 $\mathrm{M}$ & Upto 100 M \\
Multiple tag Reading & less then thousand tags within 3 M of Reader range & More then 1000 tags recognized upto 100 mph \\
\hline
\end{tabular}

RFID systems are typically used for object tracking and identification purposes. The system application accessed through Reader can perform data processing for onward usage in a range of applications like: Asset Tracking, Race Timing, E-Passport, Transportation, Payments, Human Implants, Supply-Chain-Management, Fleet and Asset-Management, Security Access-Control, E-Commerce, and Traffic Analysis and Management [9,11-14]. The IoT-enabled RFID system facilitates all such systems without any physical exposure and in bulk. However, such facilities come with security threats because of the underlying wireless media used for the communication between the tag and the Reader [15-17]. To make an RFID system acceptable and meet industrial standards, the following security features should be considered during the design phase of RFID security schemes:

1. The security scheme should preserve user privacy and anonymity.

2. The scheme should ensure forward and backward secrecy. 
3. The scheme should prevent insider attacks and replay attacks.

4. The system should have capabilities to withstand impersonation and forgery attacks.

5. The system should provide mutual authentication and thwart man in middle attack.

6. The system should be user-friendly and should have the provision of updation and alteration of tag data at any time.

Various authentication protocols have been proposed for securing RFID systems [3,12,13,17-33]. Some of these protocols are based on public key infrastructure (PKI) $[12,18,19,32,33]$. Due to the resource-constrained nature of RFID, the protocols based on PKI are unenviable. Some of the schemes have been proposed on lightweight cryptographic primitives. However, many such schemes based on merely the lightweight primitives were proved as insecure [23,27,29-31].

In 2005, Yang et al. proposed an RFID authentication protocol based on only exclusive-OR (XOR) and hash functions [23]. Some other protocols were also proposed in [21,26,30], using only lightweight hash, XOR and/or symmetric encryption. Despite their $[21,23,26,30]$ claims to provide flawless security, Piramuthu [28] proved that the protocols in [23,30] are vulnerable to replay attack, the protocol in [26] is vulnerable to impersonation of the tag and the Reader, and protocol proposed by Cai et al. [30] is vulnerable to denial-of-service (DoS) and impersonation of tag. Cho et al. [31] then proposed another hash-based protocol for securing RFID. However, Safkhani et al. [29], through cryptanalysis, proved that Cho et al.'s protocol is insecure against DoS, as well as impersonation attacks. Another authentication protocol for securing RFID systems using only symmetric key operations was proposed by Ayaz et al. [17]. However, in their protocol [17], the authentication is performed on the basis of biometrics verification. Such biometric verification may not be desirable in many scenarios, like anti-counterfeiting of life saving drugs, recording and counting number of specific goods moving in and out of a store, etc.

\subsection{Motivations and Contributions}

Quite recently, Gope and Hwang [3] argued that the existing protocols [21,23,26,29-31] based on hash functions are impractical. Then Gope and Hwang presented a new lightweight authentication protocol using only hash functions. They claimed to avoid all known attacks while maintaining efficiency. However, in this paper, we show that the protocol of Gope and Hwang is vulnerable to collision, DoS, and stolen verifier attacks. Moreover, this article presents an improved and robust protocol using only lightweight symmetric cryptography primitives for IoT-based RFID systems to resist all known attacks. The general contributions of this article include:

- Cryptanalysis of the baseline [3] protocol.

- Proposed an improved authentication protocol using only lightweight symmetric key primitives to overcome the security issues of the baseline protocol.

- Performed formal and informally security analysis of the proposed protocol.

- Solicited the comparison of the proposed protocol with related existing protocols with respect to security features.

- Accomplished the comparison of the proposed protocol with related existing protocols with respect to performance, including communication, as well as computation complexity.

\subsection{Adversarial Model}

The proposed protocol is designed keeping in mind the following adversarial model where common assumptions as pointed out in [34] are made. The following assumptions are considered as the capabilities of the adversary $\mathcal{A}$.

1. The public channel is under full control of $\mathcal{A}$, so that the $\mathcal{A}$ can intercept, revert, modify, replay, or even send a fresh fabricated message.

2. $\mathcal{A}$ has the capability to extract some of the information of the tag by power analysis. However, shared key of the tag and Server is secret and is inaccessible to any adversary. 
3. $\mathcal{A}$ can be any deceitful tag or an outsider of the system.

4. The database attached to the Server is inaccessible, and no adversary $\mathcal{A}$ can access the private key of the Server.

\subsection{Road Map}

The rest of the article is organized in various sections. In Section 1.4, a brief overview of the protocol of Gope and Hwang [3] is presented. Section 2 presents the proposed protocol, whereas Section 3 presents the detail security analysis of the proposed protocol. Section 4 presents the comparative analysis of the proposed protocol with existing protocols, and, finally, Section 5 concludes the article.

\subsection{Review of Baseline Protocol}

This section first reviews the baseline protocol of Gope and Hwang's [3] and then performs its cryptanalysis. Table 2 presents some of the notations used in the baseline protocol. The proposed protocol designed for RFID consists three main entities: (1) Database Server, (2) Reader Device, and, (3) RFID tags. The network layout of the RFID System divided into several RFID clusters. Every cluster consists of a Reader and many tags. Tags can shift from one cluster to another. Every Reader of the cluster authenticates the registered tags through the Database Server. Each Reader and Database Server share a symmetric key $K_{r s}$ [3]. Gope and Hwang's [3] authentication scheme consists of two main phases: (1) tag Registration Phase and (2) tag Authentication Phase.

Table 2. Notation Guide.

\begin{tabular}{ll}
\hline Notations & Description \\
\hline$T$ & RFID-tag \\
$R$ & Reader Device \\
$S$ & Database Server System \\
$I D_{T_{i}}$ & ith tag identity \\
$A I D_{T}$ & One-time tag alias identity \\
$S I D$ & Shadow identity \\
$R_{j}$ & jth Reader identity \\
$N_{t}$ & tag Random number \\
$N_{r}$ & Reader Random number \\
$K_{t s}$ & Shared key of Server and tag \\
$K_{e m g}$ & Shared emergency key of Server and tag \\
$K_{r s}$ & Server and Reader shared secret key \\
$\operatorname{Tr}_{s e q}$ & Track sequence number (used by both S and T) \\
$r_{j}$ & Randomly derived from Shadow-ID and Emergency Key \\
$h()$. & Hash function \\
$\oplus$ & The exclusive XOR operation \\
$\|$ & concatenation \\
\hline
\end{tabular}

\subsection{Baseline Protocol Tag Registration Phase}

The following steps, as shown in Figure 2, are performed for tag registration:

Step BLR 1: $\operatorname{tag}_{\mathbf{i}} \stackrel{\mathbf{I D}_{\mathrm{T}_{\mathbf{i}}}}{\longrightarrow} \mathbf{S}$

Each tag $\left(\operatorname{tag}_{i}\right)$ submits $I D_{T_{i}}$ to the Server $S$.

Step BLR 2: $\mathbf{S} \stackrel{\mathbf{M}}{\longrightarrow} \mathbf{t a g}_{\mathbf{i}}:\left\langle\mathbf{M}=\left\{\mathbf{K}_{\mathbf{t s}},\left(\mathbf{S I D}, \mathbf{K}_{\mathbf{e m g}}\right), \mathbf{T r}_{\mathbf{s e q}}, \mathbf{h}().\right\}\right\rangle$

$S$ generates random number $n_{s}$ and computes $K_{t s}=h\left(I D_{T_{i}} \| n_{S} \oplus I D_{s}\right) . S$ then generates a set of unlikeable shadow identities $I D_{s}$, and $S I D=\left\{s i d_{1}, s i d_{2} \cdots\right\}$, where the $s i d_{j} \in S I D$. $S$ computes $s i d_{j}=h\left(I D_{T_{i}}\left\|r_{j}\right\| K_{t s}\right)$. Further, $S$ generates a set of emergency keys $K_{e m g}=\left\{k_{e m g_{1}}, k_{e m g_{2}} \cdots\right\}$, each of the keys corresponding to specific $s_{i} d_{j} \in S I D$, where each $k_{e m g_{i}} \in K_{e m g}$. $S$ then computes $k_{e m g_{i}}=h\left(I D_{T_{i}}\left\|s i d_{j}\right\| r_{j}\right)$. Then $S$ generates a 32-bit random sequence number $T r_{s e q}$ and random 
number $m$ and matches it with $T r_{s e q}, T r_{s e q}=m$. $S$ then sends the $T r_{s e q}$ to the $t a g_{i}$ through Reader $R_{i}$ by maintaining the copy of $T r_{s e q}$ in its database for speeding up the authentication process. $S$ authenticates the validity of RFID tag $I D_{T_{i}}$ based on $T R_{s e q}$. If $T R_{s e q}$ does not have a match within the record of $S$, it terminates the process. In this case, the RFID tag $I D_{T_{i}}$ will use one of its fresh pair of the emergency key $k_{e m g_{j}} \in K_{e m g}$ and shadow ID $s_{i d} \in S I D$. The used pair of shadow ID and emergency ID (SID, $K_{\text {emg }}$ ) must be deleted from both, the Database Server $S$ and the RFID tag $I D_{t_{i}}$. Database Server $S$ again updates and send $\left\{K_{t s},\left(S I D, K_{e m g}\right), T r_{s e q}, h().\right\}$ through a secure channel for further communication.

Step BLR 3: $\operatorname{tag}_{i}$, upon receiving message from $S$, stores $\left\{I D_{T_{i}}, K_{t s},\left(S I D, K_{e m g}\right), T r_{s e q}, h().\right\}$ in its memory.

\begin{tabular}{|c|c|c|}
\hline $\operatorname{Lag}_{i}\left\{I D_{T_{i}}\right\}$ & & Database Server $\{S\}$ \\
\hline \multirow[t]{2}{*}{ Identity: $I D_{T_{i}}$} & $M=\left\{I D_{T_{i}}\right\}$ & \\
\hline & $M=\left\{I D_{T_{i}}, K_{t s}, T r_{\text {seq }},\left(S I D, K_{\text {emg }}\right)\right\}$ & $\begin{array}{l}\text { Generate: } n_{s} \\
\text { Generate random number: } m \\
\text { Set: } \operatorname{Tr}_{s e q}=m \\
\text { Compute: } K_{t s}=h\left(I D_{T_{i}} \| n_{s}\right) \oplus I D_{s} \\
\text { sid } d_{j}=h\left(I D_{T_{i}} \mid\|r\| K_{t s}\right. \\
\text { emg } g_{j}=h\left(I D_{T_{i}}\left\|s i d_{j}\right\| r_{j}\right. \\
\text { sid } d_{j} \in S I D, K_{e m g j} \in K_{e m g} \\
\text { Store: }\left\{I D_{T_{i}}, K_{t s}, T r_{s e q},\left(S I D, K_{e m g}\right)\right\}\end{array}$ \\
\hline $\begin{array}{l}\text { Store: } \\
\left\{I D_{T_{i}}, K_{t s}, \operatorname{Tr}_{\text {seq }},\left(S I D, K_{\text {emg }}\right)\right\}\end{array}$ & & \\
\hline
\end{tabular}

Figure 2. Gope-Hwang's proposed registration scheme.

\subsection{Baseline Protocol Tag Authentication Phase}

The registered tag initiates the authentication process, as shown in Figure 3, and is detailed as follows:

Step BLA 1: $\operatorname{tag}_{\mathbf{i}} \stackrel{\mathbf{M}_{\mathrm{A}_{1}}}{\longrightarrow} \mathbf{R}_{\mathbf{i}}:\left\langle\mathbf{M}_{\mathbf{A}_{\mathbf{1}}}=\left\{\mathbf{A I D}_{\mathbf{T}}, \mathbf{N}_{\mathbf{x}}, \mathbf{T r}_{\mathbf{s e q}}, \mathbf{V}_{\mathbf{1}}\right\}\right\rangle$

$\operatorname{tag}_{i}$ with identifier $I D_{T_{i}}$ generates random number $N_{t}$, and derives $A I D_{T}=$ $h\left(I D_{T_{i}}\left\|K_{t s}\right\| N_{t} \| T r_{s e q}\right), N_{x}=K_{t s} \oplus N_{t}$. The tag then computes $V_{1}=h\left(A I D_{t i}\left\|K_{t s}\right\| N_{x} \| R_{i}\right)$ and sends message request as $M_{A_{1}}$ to the Reader device $R_{i}$. $R_{i}$ also receives a recently used sequence number from $S$ for mutual authentication. In the case of synchronization loss, the tag uses one of its fresh pair $\left(s i d_{j}, K_{e m g_{j}}\right)$. Subsequently, it is assigned to the $s i d_{j}$ as $A I D_{T}$ and then $k_{e m g_{j}}$ as $K_{t s} . t_{a g}$ sends $M_{A_{1}}$ to the Reader $R_{i}$.

Step BLA 2: $\quad \mathbf{R}_{\mathbf{i}} \stackrel{\mathbf{M}_{\mathbf{A}_{2}}}{\longrightarrow} \mathbf{S}:\left\langle\mathbf{M}_{\mathbf{A}_{2}}=\left\{\mathbf{N}_{\mathbf{y}}, \mathbf{R}_{\mathbf{i}}, \mathbf{V}_{\mathbf{2}}, \mathbf{M}_{\mathbf{A}_{\mathbf{1}}}\right\}\right\rangle$

Upon receiving request from $t a g_{i}$, Reader $R_{i}$ of the $i^{\text {th }}$ cluster (in which $t a g_{i}$ is located) generates random number $N_{r}$ and computes $N_{y}=K_{r s} \oplus N_{r}, V_{2}=h\left(M_{A_{1}}\left\|N_{r}\right\| K_{r s}\right) . R_{i}$ then sends $M_{A_{2}}$ to $S$ for verification.

Step BLA 3: $\mathbf{S} \stackrel{\mathbf{M}_{\mathbf{A}_{3}}}{\longrightarrow} \mathbf{R}_{\mathbf{i}}:\left\langle\mathbf{M}_{\mathbf{A}_{3}}=\left\{\mathbf{T}_{\mathbf{r}}, \mathbf{V}_{3}, \mathbf{V}_{\mathbf{4}}, \mathbf{x}_{\text {(ifreq. }}\right\}\right\rangle$

When $S$ receives a request from $R_{i}$, first it validates the track sequence number $T r_{\text {seq }}$ by computing $V_{1}=h\left(A I D_{T}\left\|K_{t s}\right\| N_{x} \| R_{i}\right) . S$ then derives $N_{t}=K_{t s} \oplus N_{x}$ and verifies $A I D_{T}$. Upon successful verification of $A I D_{T}, S$ generates a random number $m$ and assigns it to $T_{r_{\text {seq }}}=m$. S also computes $T_{r}=h\left(K_{t s}\left\|I D_{T_{i}}\right\| N_{t}\right) \oplus T_{r_{s e q}}, V_{4}=h\left(T_{r}\left\|K_{t s}\right\| I D_{T_{i}} \| N_{t}\right), V_{3}=h\left(R_{i}\left\|N_{r}\right\| K_{r s}\right)$ to create a message $M_{A_{3}}$ and the $S$ sends $M_{A_{3}}$ to $R_{i}$. Finally, $S$ computes $K_{T_{\text {new }}}=h\left(K_{t s}\left\|I D_{T_{i}}\right\| T_{\text {seq new }}\right)$ and updates $K_{T s_{\text {new }}}$ and $T r_{\text {seqnew }}$. In case the message $M_{A_{1}}$ does not contain $T_{r_{\text {seq }}}$, then $S$ randomly generates a new shared key $K_{T S_{\text {new }}}$ using the emergency key $K_{e m g_{j}}$ and real identity of the tag $I D_{T_{i}}$. Then $x=K_{t s_{\text {new }}} \oplus h\left(I D_{T_{i}} \| K_{\text {emg }}\right)$ is computed and $x$ is sent with the message $M_{A_{3}}$, where $V_{4}$ is calculated as $V_{4}=h\left(N_{t}\left\|T_{r}\right\| x \| K_{e m g_{j}}\right)$. 
Step BLA 4: $\quad \mathbf{R}_{\mathbf{i}} \stackrel{\mathbf{M}_{\mathbf{A}_{4}}}{\longrightarrow} \operatorname{tag}_{\mathbf{i}}:\left\langle\mathbf{M}_{\mathbf{A}_{4}}=\left\{\mathbf{T}_{\mathbf{r}}, \mathbf{V}_{\mathbf{4}}, \mathbf{x}_{\text {(ifreq. }}\right\}\right\rangle$

$R_{i}$ receives $M_{A_{3}}$ and computes $h\left(R_{i}\left\|N_{r}\right\| K_{r s}\right)$, and validates if it is equal to $V_{3}$. Upon successful validation, $R_{i}$ sends $M_{A_{4}}$ to $\operatorname{tag}_{i}$. Contrarily, the Reader $R_{i}$ terminates the session.

Step BLA 5: $\quad \operatorname{tag}{ }_{i}$, on receiving $M_{A_{4}}$, computes $h\left(\operatorname{Tr}\left\|K_{t s}\right\| I D_{T_{i}} \| N_{t}\right)$ and verifies its equality with $V_{4}$. Upon success, $t a g_{i}$ derives $K_{t s_{\text {new }}}=h\left(K_{t s}\left\|I D_{T_{i}}\right\| T r_{\text {seq new }}\right)$ and stores $K_{t s}=K_{t s_{\text {new }}}, \operatorname{Tr}_{\text {seq }}=T r_{\text {seqnew }}$ for future communication.

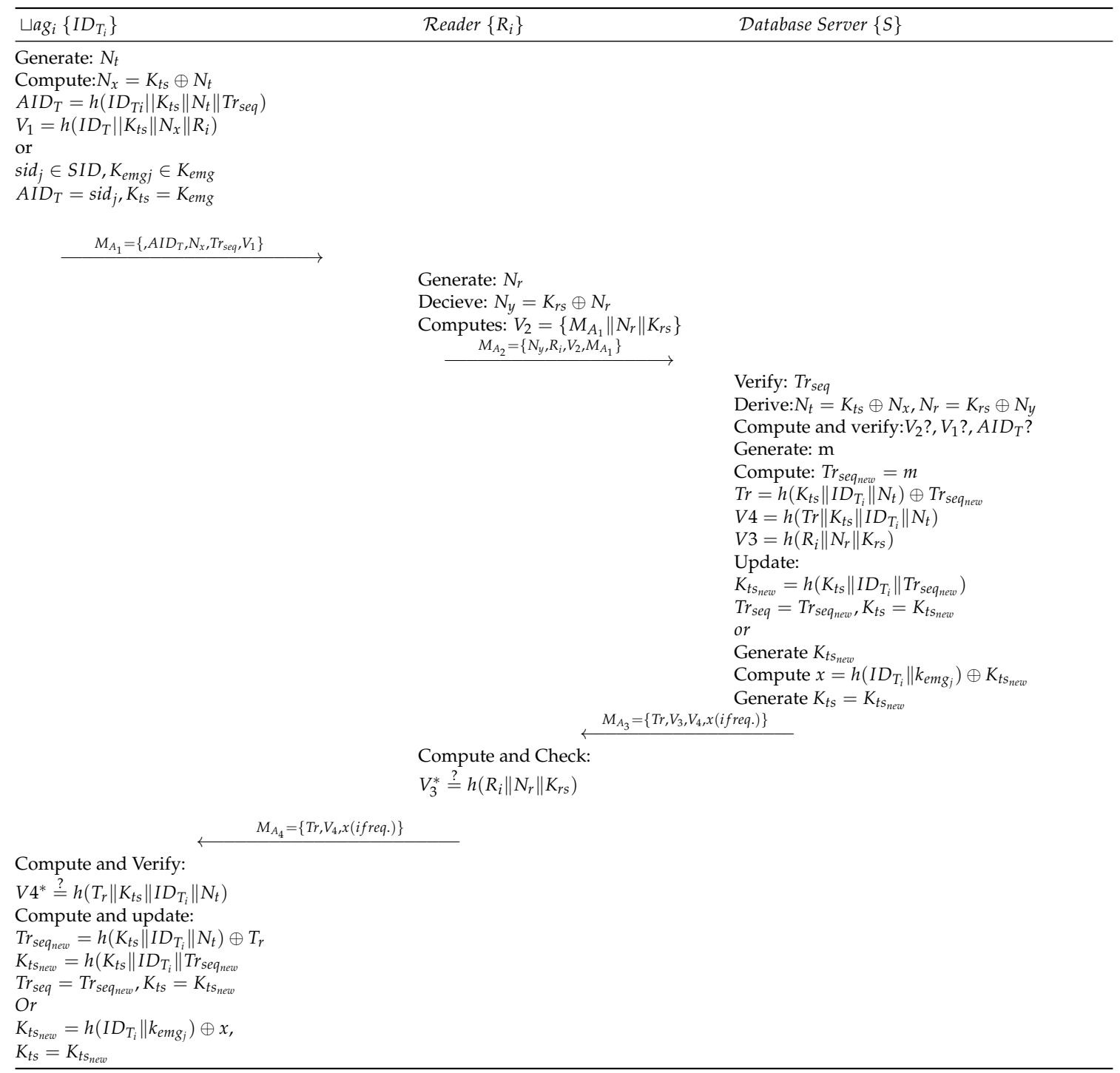

Figure 3. Gope-Hwang's proposed authentication scheme.

\subsection{Cryptanalysis of Baseline Protocol}

The following subsections show that the baseline protocol is vulnerable to: (1) Collision, (2) Stolen Verifier, and (3) DoS Attacks.

\subsubsection{Vulnerable to Collision Attack}

The correctness of the baseline protocol [3] depends on Track sequence number $\operatorname{Tr}_{\text {seq }}$, generated randomly during registration and saved in the database, as well as in the tag's memory. This number $T r_{\text {seq }}$ is sent in authentication request $M_{A_{1}}=\left\{A I D_{T}, N_{x}, T r_{\text {seq }}, V_{1}\right\}$ by the tag and then, upon reception of $M_{A_{1}}$, the Reader $R_{i}$ sends $M_{A_{2}}=\left\{M_{A_{1}}, N_{y}, R_{i}, V_{2}\right\}$ to the Database Server. $S$ verifies the legitimacy of $\operatorname{Tr}_{\text {seq }}$ by comparing it with the one stored in its database. The randomness can cause two or more 
track sequence numbers to have the same value (collision), and there is no mechanism to handle such collisions. Then the process will terminate abnormally, and the legitimate tag will be deprived of its right to authentication and access.

\subsubsection{Vulnerable to Stolen Verifier Attack}

Considering the common adversarial modal, as mentioned in Section 1.2, an adversary can steal the verifier table stored unencrypted on server. $\mathcal{A}$ based on the track sequence number $\operatorname{Tr}_{\text {seq }}$ and the public request from any of the previous session $M_{A_{1}}:\left\{A I D_{T}, N_{x}, T r_{\text {seq }}, V_{1}\right\}$ and $M_{A_{2}}$ : $\left\{N_{y}, R_{i}, V_{2}, M_{A_{1}}\right\}$ can then generate login request using the previous session's $N_{x}, N_{y}$ and the stolen new $T r_{\text {seq. }}$. The request will pass the authentication test as all values are valid. Hence baseline protocol is also vulnerable to stolen verifier attack.

\subsubsection{Vulnerable to DoS Attack}

In the baseline proposal [3], an adversary can launch a DoS attack by continuously generating 32 bits random $T r_{\text {seq }}$ numbers and send it to the Database Server. It will keep $S$ busy in verifying dummy random numbers, thus restricting $S$ to serve a legitimate request.

\section{Proposed Scheme}

Like the baseline protocol, the proposed protocol for RFID consists of three main entities: (1) Database Server, (2) Reader Device, and (3) RFID tags. The network layout of the RFID System is divided into several RFID clusters. Every cluster consists of a Reader and many tags. Tags can shift from one cluster to another. Every Reader of the cluster authenticates the registered tags through the Database Server. Each Reader and Database Server share a symmetric key $K_{r s}$. Proposed improved authentication scheme consists of two main phases; (1) tag Registration Phase, (2) tags Authentication Phase.

\subsection{Tags Registration Phase}

The following steps, as shown in Figure 4, are performed for tag registration:

Step PTR 1: $\operatorname{tag}_{\mathbf{i}} \stackrel{\mathbf{I D}_{\mathrm{T}_{i}}}{\longrightarrow} \mathbf{S}$

Each tag submits $I D_{T_{i}}$ to the Server $S$.

Step PTR 2: $\mathbf{S} \stackrel{\mathbf{M}}{\longrightarrow} \operatorname{tag}_{\mathbf{i}}:\left\langle\mathbf{M}=\left\{\mathbf{I D}_{\mathbf{T}_{\mathbf{i}}}, \mathbf{K}_{\mathbf{t s}}, \mathbf{A I D}\right\}\right\rangle$

$S$ generates a random number $n_{S}$ and computes $K_{t s}=h\left(I D_{T_{i}} \| n_{S} \oplus I D_{S}\right)$. S generates $r_{i}$ randomly and computes one-time alias $\operatorname{tag}_{i}{ }^{\prime}$ s identity $A I D=E_{S_{x}}\left(I D_{T_{i}} \| r_{T_{i}}\right)$ by encrypting it with the Secret Key $s_{x}$ of $S . S$ authenticates $t a g_{i}$ based on $A I D_{T}$ in authentication phase by checking if a request is valid or not. $S$ stores and sends $M$ to the RFID tag through a secure channel.

Step PTR 3: Upon receiving the message from $S$, $\operatorname{tag}_{i}$ stores the information $M=\left\{I_{T_{i}}, K_{t s}, A I D\right\}$ in its memory.

\begin{tabular}{|c|c|c|}
\hline $\operatorname{Lag}_{i}\left\{I D_{T_{i}}\right\}$ & & Database Server $\{S\}$ \\
\hline \multirow[t]{2}{*}{ Identity: $I D_{T_{i}}$} & $M=\left\{I D_{T_{i}}\right\}$ & \\
\hline & $M=\left\{I D_{T_{i}}, K_{t s}, A I D\right\}$ & $\begin{array}{l}\text { Generate: } n_{s} \\
\text { Compute: } K_{t s}=h\left(I D_{T_{i}} \| n_{s}\right) \oplus I D_{s} \\
A I D=E_{s_{x}}\left(I D_{T_{i}} \| r_{T_{i}}\right) \\
\text { Store: }\left\{I D_{T_{i}}, K_{t s}, A I D\right\}\end{array}$ \\
\hline Store: $\left\{I D_{T_{i}}, K_{t s}, A I D\right\}$ & & \\
\hline
\end{tabular}

Figure 4. Registration phase of the proposed protocol. 


\subsection{Tags Authentication Phase}

The registered tag initiates the authentication process, as shown in Figure 5, and is detailed as follows:

Step PTA 1: $\operatorname{tag}_{\mathbf{i}} \stackrel{\mathbf{M}_{\mathrm{A}_{1}}}{\longrightarrow} \mathbf{R}_{\mathbf{i}}:\left\langle\mathbf{M}_{\mathbf{A}_{\mathbf{1}}}=\left\{\mathbf{A I D}_{\mathbf{T}}, \mathbf{N}_{\mathbf{x}}, \mathbf{T}_{\mathbf{1}}, \mathbf{V}_{\mathbf{1}}\right\}\right\rangle$

RFID tag with identifier $I D_{T_{i}}$ generates a random number $N_{t}$ and derives $N_{x}=K_{t s} \oplus N_{t}$ and $V_{1}=h\left(A I D_{t}\left\|K_{t s}\right\| N_{x} \| R_{i}\right)$. The tag then initiates an authentication request request by sending $M_{A_{1}}$ to $R_{i}$.

Step PTA 2: $\quad \mathbf{R}_{\mathbf{i}} \stackrel{\mathbf{M}_{\mathbf{A}_{2}}}{\longrightarrow} \mathbf{S}:\left\langle\mathbf{M}_{\mathbf{A}_{\mathbf{2}}}=\left\{\mathbf{N}_{\mathbf{y}}, \mathbf{R}_{\mathbf{i}}, \mathbf{V}_{\mathbf{2}}, \mathbf{M}_{\mathbf{A}_{\mathbf{1}}}, \mathbf{T}_{\mathbf{2}}\right\}\right\rangle$

Upon receiving the request from the tag, Reader $R_{i}$ of the $i^{\text {th }}$ cluster (in which tag is located) first verifies the timestamp freshness as $\left(T_{2}-T_{1}\right) \leq \Delta T . R_{i}$ generates a random number $N_{r}$ and computes $N_{y}=K_{r s} \oplus N_{r}, V_{2}=h\left(M_{A_{1}}\left\|N_{r}\right\| K_{r s} \| T_{2}\right) . R_{i}$ sends $M_{A_{2}}$ to the $S$ for verification.

Step PTA 3: $\mathbf{S} \stackrel{\mathbf{M}_{\mathbf{A}_{3}}}{\longrightarrow} \mathbf{R}_{\mathbf{i}}:\left\langle\mathbf{M}_{\mathbf{A}_{3}}=\left\{\mathbf{V}_{\mathbf{3}}, \mathbf{V}_{\mathbf{4}}, \mathbf{Z}_{\mathbf{T}}, \mathbf{T}_{\mathbf{3}}\right\}\right\rangle$

When $S$ receives the request from $R_{i}$, first it verifies $\left(T_{3}-T_{2}\right) \leq \Delta T$, then derives $N_{t}=K_{t s} \oplus$ $N_{x}$ and $N_{r}=K_{r s} \oplus N_{y}$. Further, $S$ computes and verifies $V_{1}=h\left(A I D_{T}\left\|K_{t s}\right\| N_{x} \| R_{i}\right), V_{2}=$ $h\left(M_{A_{1}}\left\|N_{r}\right\| K_{r s} \| T_{2}\right)$. Then $S$ verifies $A I D_{T_{i}}$ by decrypting it as $A I D_{T_{i}}=D_{S_{x}}\left(I D_{T_{i}} \| r_{i}\right)$. Upon successful verification, $S$ computes $V 3=h\left(R_{i}\left\|N_{r}\right\| K_{r s} \| T_{3}\right)$ and $V 4=h\left(K_{t s}\left\|I D_{T_{i}}\right\| N_{t} \| T_{3}\right)$. $S$ then updates $A I D_{T_{i}(\text { new })}=E_{S_{x}}\left(I D_{T_{i}} \| r_{i_{(n e w)}}\right)$ and computes $Z_{T}=A I D_{T_{\text {new }}} \oplus K_{T s}$. S, finally, sends $M_{A_{3}}$ to $R_{i}$.

Step PTA 4: $\quad \mathbf{R}_{\mathbf{i}} \stackrel{\mathbf{M}_{\mathbf{A}_{\mathbf{4}}}}{\longrightarrow} \operatorname{tag}_{\mathbf{i}}:\left\langle\mathbf{M}_{\mathbf{A}_{\mathbf{4}}}=\left\{\mathbf{V}_{\mathbf{4}}, \mathbf{T}_{\mathbf{4}}, \mathbf{Z}_{\mathbf{T}}\right\}\right\rangle$

Upon receiving $M_{A_{3}}, R_{i}$ checks freshness of the timestamp $\left(T_{4}-T_{3}\right) \leq \Delta T$. $R_{i}$ computes $h\left(R_{i}\left\|N_{r}\right\| K_{r s}\right)$ and verifies its equality with the received $V_{3}$. Upon success, $R_{i}$ sends $M_{A_{4}}$ to $\operatorname{tag}_{i}$. Otherwise, $R_{i}$ terminates the session.

Step PTA 5: Upon receiving $M_{A_{4}}$, $\operatorname{tag}$ first checks freshness of the timestamp and upon success verifies the message $V 4^{*} \stackrel{?}{=} h\left(K_{t s}\left\|I D_{T_{i}}\right\| N_{t}\right)$. Then $\operatorname{tag}_{i}$ computes and updates $A I D_{T_{i}(n e w)}=$ $\left(Z_{T} \oplus K_{T s}\right), A I D_{T_{i}}=A I D_{T_{i}(\text { new })}$ and saves the information for the next authentication process. 


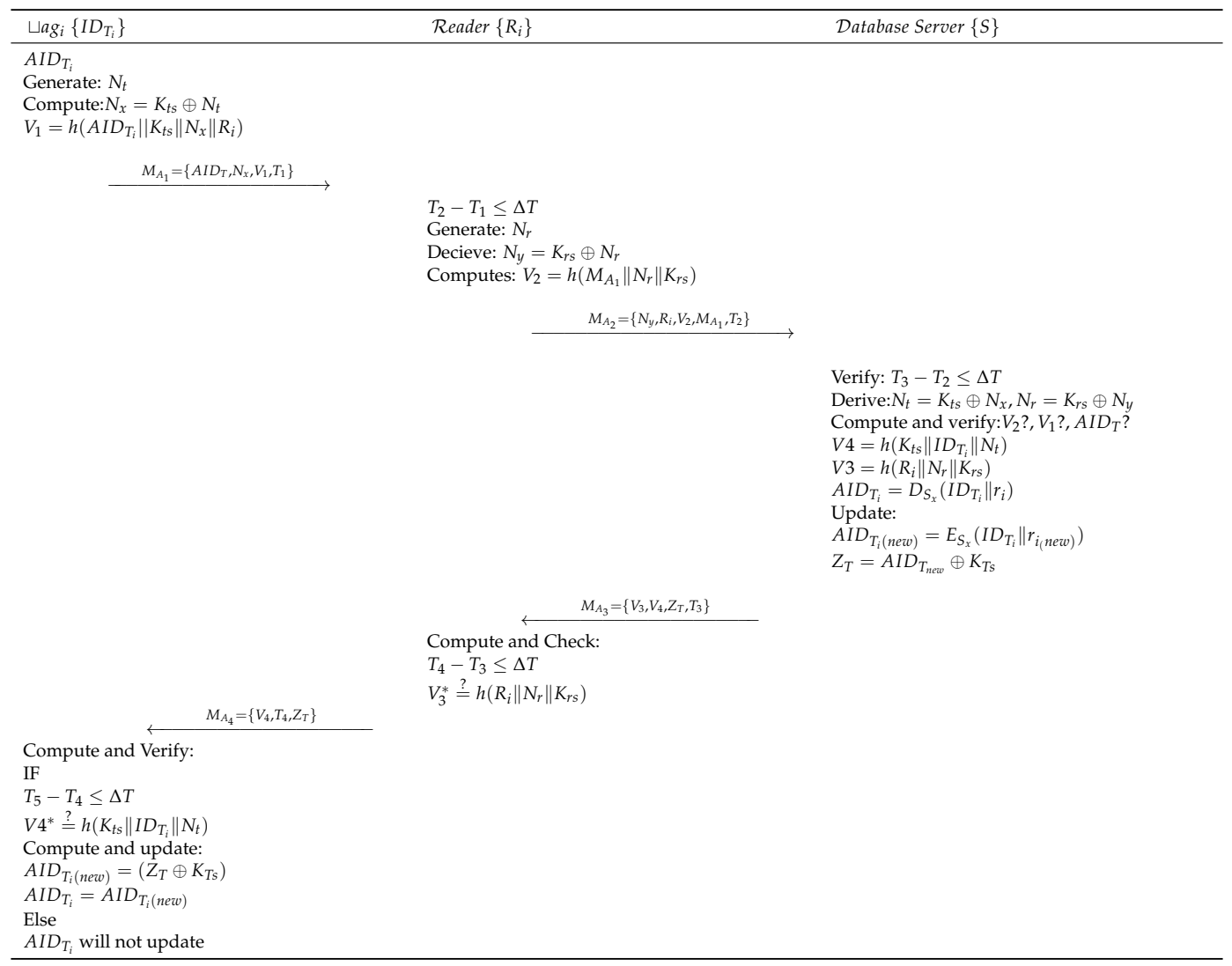

Figure 5. Proposed authentication protocol.

\section{Security Analysis}

In this section, the security analysis of the proposed protocol under the adversarial model briefed in Section 1.2 is performed. The task is accomplished by formal analysis under Burrows Abadi-Needham (BAN) logic, and informal security features are explained. Moreover, the robustness of the proposed protocol is also analyzed through the automated tool, ProVerif-a widely accepted simulation tool for verification of the security of authentication protocols [35-43].

\subsection{BAN Logic-Based Formal Security Analysis}

BAN logic consists of a set of rules that can be used to analyzed information exchange protocols. It specifically determines if the information exchanged in a protocol is resistant against eavesdropping and is trustworthy and secured. The mutual authentication of the proposed protocol has been checked using the BAN logic [44]. Different rules of BAN logic, including idealized form, assumptions, and proofs, are shown in Table 3. 
Table 3. BAN logic Notations.

\begin{tabular}{ll}
\hline Notations & Description \\
\hline$P \mid \equiv X$ & P believes that $\mathrm{X}$ \\
$P \triangleleft X$ & P sees that $\mathrm{X}$ \\
$P \mid \sim X$ & P once said $\mathrm{X}$ \\
$P \Rightarrow X$ & P have total jurisdiction on $\mathrm{X}$ \\
$\#(X)$ & $\mathrm{X}$ is updated and fresh \\
$(X, Y)$ & $\mathrm{X}, \mathrm{Y}$ is component of formula $(\mathrm{X}, \mathrm{Y})$ \\
$<X>Y$ & $\mathrm{X}$ is combine with $\mathrm{Y}$ \\
$(X)_{K}$ & Hash of message $\mathrm{X}$ using a key $\mathrm{K}$ \\
$P \stackrel{K}{\longrightarrow} Q$ & P and $\mathrm{Q}$ share key $\mathrm{K}$ for communication \\
$A I D T_{i}$ & $A I D T_{i}$ is one time session key \\
$\frac{P \mid \equiv P \stackrel{K}{P} \longrightarrow Q \cdot p \triangleleft<X>_{K}}{P|\equiv Q| \sim X}$ & Message-Meaning rule \\
$\frac{P \mid \equiv \#(X)}{P \mid \equiv \#(X, Y)}$ & Freshness-conjuncatenation rule \\
$\frac{P|\equiv \#(X), P| \equiv Q \mid \sim X}{P|\equiv Q| \equiv X}$ & Nonce-verification rule \\
$\frac{P|\equiv Q \Rightarrow X, P| \equiv Q \mid \equiv X}{P \mid \equiv X}$ & Jurisdiction rule \\
$P \mid \equiv X$ & P believes $\mathrm{X}$ \\
\hline
\end{tabular}

To analyze the security of a protocol using BAN logic, different goals have to be determined. In the case of the proposed protocol, eight different goals have been determined based on BAN logic. These goals are shown in the following list.

- Goal 1: $R_{i} \mid \equiv \operatorname{tag} \stackrel{A I D_{T}}{\longleftrightarrow} \mathrm{Ri}$

- Goal 2: $R_{i}|\equiv \operatorname{tag}| \equiv \operatorname{tag} \stackrel{A I D_{T}}{\longleftrightarrow} \mathrm{Ri}$

- Goal 3: $S_{j} \mid \equiv R_{i} \stackrel{A I D_{+}}{\longleftrightarrow} \mathrm{Sj}$

- Goal 4: $S_{j}\left|\equiv R_{i}\right| \equiv R_{i} \stackrel{A I D_{T}}{\longleftrightarrow} \mathrm{Sj}$
- Goal 5: $R_{i} \mid \equiv S_{j} \stackrel{A I D_{T}}{\longleftrightarrow} \mathrm{Ri}$

- Goal 6: Ri| $\equiv S_{j} \mid \equiv S_{j} \stackrel{A I D_{T}}{\longleftrightarrow} \mathrm{Ri}$

- Goal 7: tag $\equiv R_{i} \stackrel{\text { AID }}{\longleftrightarrow}$ tag

- Goal 8: $\operatorname{tag}\left|\equiv R_{i}\right| \equiv R_{i} \stackrel{A I D_{T}}{\longleftrightarrow}$ tag.

To achieve the goals listed above, the security analysis using BAN logic has been divided into three parts. Part1 shows the idealized form of the protocol and is proved in Part3, whereas Part2 uses assumptions to analyzed the proposed protocol.

Part1: The idealized form for the proposed protocol has been discussed as follows:

- M1: $\operatorname{tag} \rightarrow$ Ri: $A I D_{T}, N_{x}:<N_{t}>_{K_{t s}}, V 1, T 1$

- M2: $R_{i} \rightarrow \mathrm{Sj}: \mathrm{M} 1, N_{y}:<N_{r}>_{K_{r s}}, R_{i}, V 2, T 2$,

- M3: $S_{j} \rightarrow \mathrm{Ri}: \mathrm{V} 3, \mathrm{~V} 4, Z_{t}:<A I D T_{i}>_{K_{\mathrm{ts}}}^{*}, \mathrm{T3}$

- M4: $R_{i} \rightarrow \operatorname{tag}: V 4, T 4, Z_{t}:\left\langle A I D T_{i}>_{K_{t s}}\right.$.

Part2: The assumptions used for analyzing the proposed protocol using BAN logic are shown below:

- A1: $\operatorname{tag} \mid \equiv \#\left(N_{t}\right)$

- A2: $R_{i} \mid \equiv \#\left(N_{r}\right)$

- A3: $S_{j} \mid \equiv \#(A I D T i)\left(r_{i}\right)$

- A4: $R_{i} \mid \equiv S_{j} \Rightarrow r_{i}$

- A5: $R_{i} \mid \equiv \operatorname{tag} \Rightarrow N_{t}$
- A6: $S_{j} \mid \equiv R_{i} \Rightarrow N_{r}$

- A7: $S_{j} \mid \equiv \operatorname{tag} \Rightarrow N_{t}$

- A8: $\operatorname{tag} \mid \equiv S_{j} \Rightarrow r_{i}$

- A9: $\operatorname{tag} \mid \equiv R_{i} \Rightarrow N_{r}$.

Part 3: Analysis of Idealized form of the proposed protocol that has been derived on the basis of BAN logic assumptions and rules is described as follows: M1: $\operatorname{tag} \rightarrow$ Ri: $A I D T_{i}, N_{x}:<N_{t}>_{K_{t s}}, T 1$ is time-stamp of tag. Using the seeing rule, the following can be achieved:

- S1: $R_{i} \triangleleft A I D T_{i}, S I D, N_{x}:<N_{t}>_{K_{t s}}, T 1$. 
According to the message-meaning rule and S1, the following can be obtained:

- S2: $R_{i}|\equiv \operatorname{tag}| \sim N_{t}$.

Using the freshness-conjuncatenation rule and S2 will achieve the following:

- S3: $R_{i}|\equiv \operatorname{tag}| \equiv N_{t}$.

Using the jurisdiction rule and $\mathrm{S} 3$, the following can be achieved:

- S4: $R_{i} \mid \equiv N_{t}$.

Using $S 4$ and the session key rule, the following can be achieved:

- S5: $R_{i} \mid \equiv \operatorname{tag} \stackrel{A I D T_{i}}{\longleftrightarrow} \operatorname{Ri}$ (Goal 1).

Using the nonce-verification rule, the following is obtained:

- S6: $R_{i}|\equiv \operatorname{tag}| \equiv \operatorname{tag} \stackrel{A^{A I D T_{i}}}{\longleftrightarrow} \mathrm{Ri}$ (Goal 2).

M2: $R_{i} \rightarrow S_{j}: M 1, N_{y}:<N_{r}>_{K_{r s}}, T 2, V 2$, whereas T2 is time-stamp of $R_{i}$.

By using the seeing rule, we achieve:

- S7: $S_{j} \triangleleft M 1, N_{y}:<N_{r}>_{K_{r s}}, T 2, V 2$.

By the message-meaning rule and S7, the following can be achieved:

- S8: $S_{j}\left|\equiv R_{i}\right| \sim N_{r}$.

By the freshness-conjuncatenation rule and $\mathrm{S8}$, the following can be computed:

- S9: $S_{j}\left|\equiv R_{i}\right| \equiv N_{r}$.

By applying the jurisdiction rule and S9, the following can be obtained:

- S10: $S_{j} \mid \equiv N_{r}$.

Using the S10 and the SK rule, the following can achieved:

- S11: $S_{j} \mid \equiv R_{i} \stackrel{A I D T_{i}}{\longleftrightarrow} \mathrm{Sj}$ (Goal 3).

Using the nonce-verification rule and S11, the following can be achieved:

- S12: $S_{j}\left|\equiv R_{i}\right| \equiv R_{i} \stackrel{A I D T_{i}}{\longleftrightarrow} \mathrm{Sj}$. (Goal 4).

M3: $S_{j} \rightarrow$ Ri: $V 3, V 4, Z t<A I D T_{i_{\text {new }}}>_{K_{t s}}^{*}, T 3, T 3$ is time-stamp of $S_{j}$.

By the seeing-rule, the following can be achieved:

- S13: $R_{i} \triangleleft V 3, V 4, Z t<A I D T_{i_{\text {new }}}>_{K_{t s}}^{*}, T 3$.

By the message-meaning rule and $\mathrm{S} 13$, the following can be obtained:

- S14: $R_{i}\left|\equiv S_{j}\right| \sim A I D T_{i_{\text {new }}}$.

By S14 and the freshness-conjuncatenation rule, the following can achieved:

- S15: $R_{i}\left|\equiv S_{j}\right| \equiv A I D T_{i_{\text {new }}}$.

By the assumption S15 and jurisdiction rule, the following can be achieved:

- $\mathrm{S} 16: R_{i} \mid \equiv A I D T_{i_{\text {new }}}$.

Using S16 and the session-key rule, the following can be achieved: 
- S17: $R_{i} \mid \equiv S_{j} \stackrel{A I D T_{\text {inew }}}{\longleftrightarrow}$ Ri. (Goal 5).

Applying nonce-verification rule, the following can be computed:

- S18: $R_{i}\left|\equiv S_{j}\right| \equiv S_{j} \stackrel{A I D T_{\text {inewo }}}{\longleftrightarrow}$ Ri. (Goal 6).

M4: $R_{i} \rightarrow \operatorname{tag}: V 4, Z t<A I D T_{i_{\text {new }}}>_{K_{t s}}, T 4, T 4$ is timestamp of $R_{i}$.

Using the seeing rule, the following can be computed:

- S19: $\operatorname{tag} \triangleleft V 4, Z t<A I D T_{i_{\text {new }}} \geq>_{t s}, T 4$.

Using the message-meaning rule and S19, the following is achieved:

- S20: $\operatorname{tag}\left|\equiv R_{i}\right| \sim A I D T_{i_{\text {new }}}^{\prime}$.

Using S20 and the freshness-conjuncatenation rule, the following can be obtained:

- $\mathrm{S} 21: \operatorname{tag}\left|\equiv R_{i}\right| \equiv A I D T_{i_{\text {newo }}}$.

Using the jurisdiction rule and S21, the following can be achieved:

- S22: $\operatorname{tag} \mid \equiv A I D T_{i_{\text {new }}}$.

Using the session-key rule, the following can be obtained:

- S23: $\operatorname{tag} \mid \equiv R_{i} \stackrel{A I D T_{\text {inerw }}}{\longleftrightarrow} \operatorname{tag}$ (Goal 7).

Finally, using the nonce-verification rule, the following can be achieved, which is also the final goal of the proposed protocol:

- S24: $\operatorname{tag}\left|\equiv R_{i}\right| \equiv R_{i} \stackrel{A I D T_{\text {inew }}}{\longleftrightarrow} \operatorname{tag}$ (Goal 8).

Consequently, using the BAN logic, it has been shown that $\operatorname{tag}, R_{i}$, and $S_{j}$ achieve mutual authentication successfully and securely attain the session key agreement.

\subsection{Security Analysis with ProVerif}

Based on applied $\pi$ calculus, ProVerif uses automated reasoning to test the security features of authentication protocols. Specifically, ProVerif can verify the reachability, correspondence, and observational equivalence, as well as secrecy properties. ProVerif supports primitive cryptographic operations [45], including MAC, digital signatures, encryption/decryption, elliptic curve operations, hash, and other functions [46]. The steps of the proposed scheme, as illustrated in Section 2 and shown in Figures 4 and 5, are simulated in ProVerif. The formal security validation model of ProVerif consists of three phases: (1) Declaration, as coded in Figure 6A, declares the constants, names, variablesm, and cryptographic function, (2) Process part, as shown in Figure 6B, defines the three processes, each for tag, Reader, and Server, and (3) Main, as implemented in Figure 6C, simulates the actual protocol. 


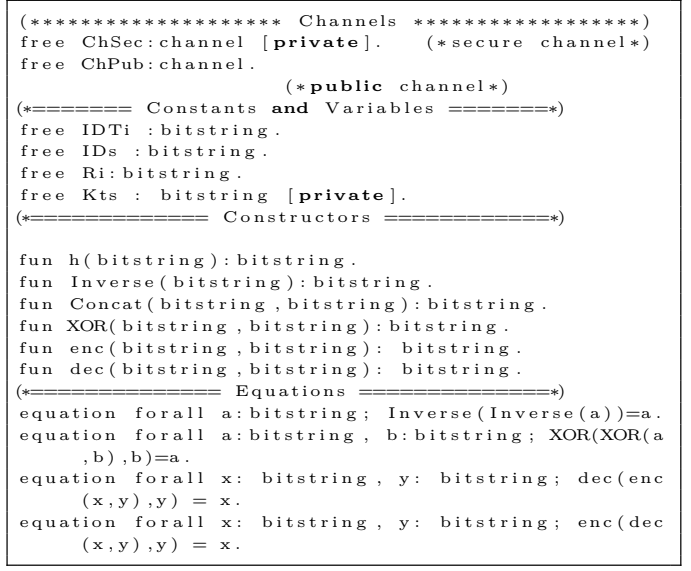

(A) Declarations
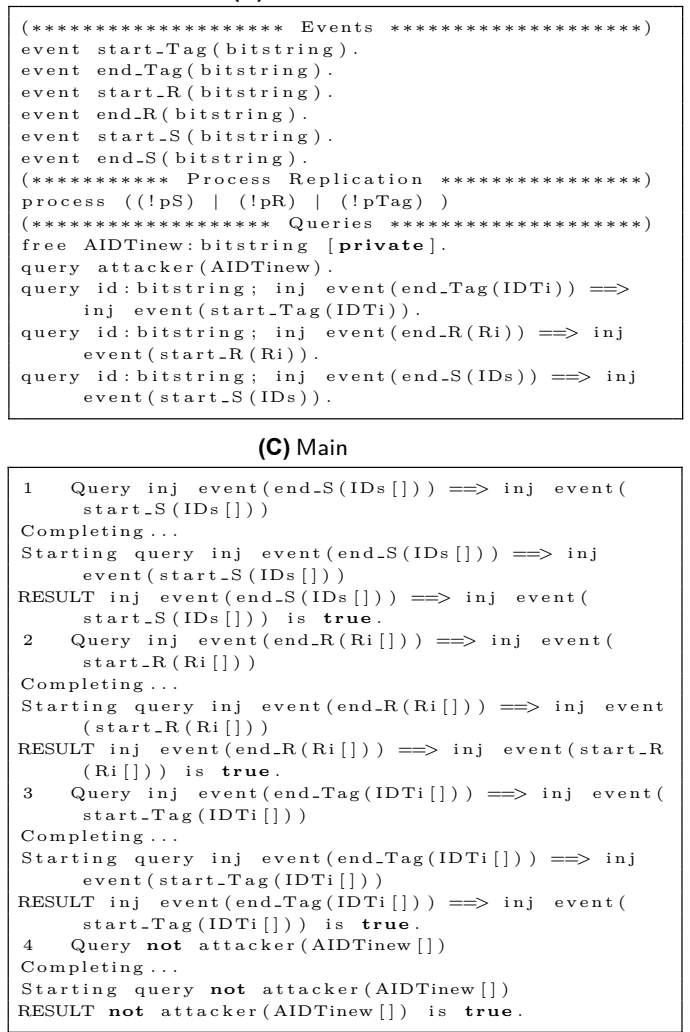

(D) Simulation Results

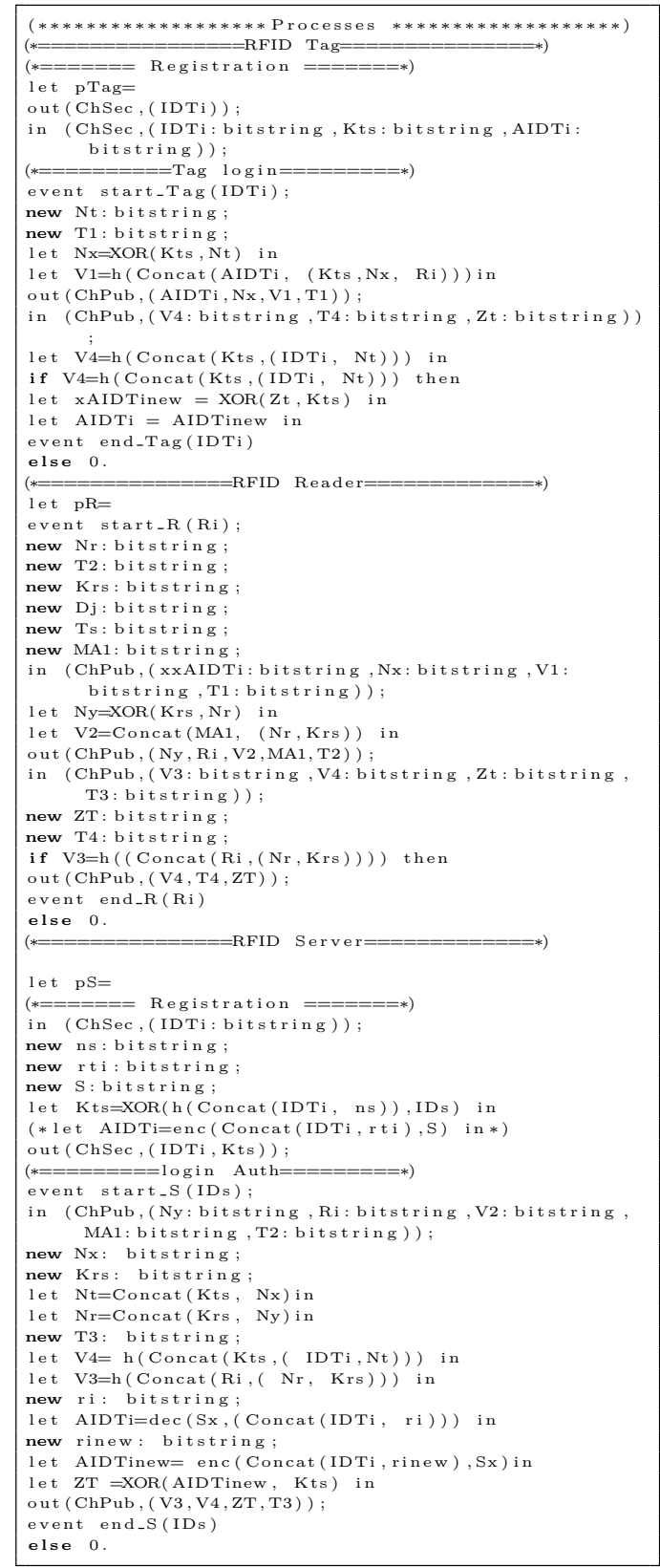

(B) Processes

Figure 6. ProVerif Simulation.

Simulation of three processes executed in parallel is performed, along with six events to validate the reachability properties of three processes. Finally, four queries are implemented. The results are shown in Figure 6D. Based on the above description of results 1, 2, and 3, all three original processes of the proposed protocol successfully started and terminated. Result 4 shows that the session tag identity $A I D_{T_{i}}$ is safe from any adversary attack. Therefore, the proposed protocol possesses correctness and provides tag secrecy.

\subsection{Informal Security Analysis}

The proposed protocol for RFID System is analyzed for security loopholes against the known attacks in the following subsections. 


\subsubsection{Mutual Authentication Between Tag And Server}

The RFID Server authenticates RFID tag by verifying a one time alias $A I D_{T_{i}}$ and $V_{1}=h\left(I D_{T}\left\|K_{t s}\right\| N \| R_{i}\right)$ in the message $M_{1}$. Only a legitimate RFID tag can form a valid request message $M_{1}$, including both these parameters, as valid $A I D_{T_{i}}$ is only known to legal tag; moreover, $I D_{T}, K_{t s}$ are known to the legal tag only. On other side, the RFID tag can authenticate the legitimacy of the Server using parameters $V_{4}$ and message $M_{3}$ in $M_{4}$. This way, the proposed protocol achieves mutual authentication property.

\subsubsection{Anonymity}

One of the basic principles of security is that an authentication protocol must not reveal the identity information of any participant (user or device) to an adversary. Anonymity is an essential factor of a secure protocol. A secure scheme guards the personal information of a user so that an adversary or intruder cannot access any information that may lead to a security breach of the system. In the proposed protocol, strong anonymity has been achieved. In the registration phase, the RFID tag registered itself with the Server $S$ through RFID-Reader using a secure channel, $M=\left\{I D_{T_{i}}, K_{t s}, A I D\right\}$.

In the login and authentication phase of the proposed protocol, message $M_{A_{1}}=$ $\left\{A I D_{T_{i}}, N_{x}, V_{1}, T_{1}\right\}$ has been sent to the Server $S$ using public channel. Here, if an adversary gets the message $M_{1}$, the adversary still cannot know the identity of the RFID tag because $A I D_{T_{i}}$ is a one-time alias identity of the tag. The original identity is kept encrypted in $A I D_{T_{i}}$ and can only be decrypted by the Server using a shared secret Key $K_{t s}$. Thus, an adversary cannot reveal the RFID tag's actual identity, hence achieving anonymity for the proposed protocol.

\subsubsection{Traceability}

A genuinely secure protocol must not reveal any identifying information of the participants to an illegitimate user. The identifying information may lead to the traceability of the RFID tag. The proposed protocol does not reveal any login information of the current of or any previous sessions that lead to a security attack on the RFID system. It is achieved through the use of different random numbers at different levels, like $N_{t}, N_{r}, r_{i}$. Furthermore, a new one-time-alias identity for the RFID tag $A I D_{T i}$ has been use, making it impossible for an adversary to guess any random number and launch an attack on the RFID system. Consequently, it can be been claimed that the proposed protocol makes the RFID tag untraceable.

\subsubsection{Backward/Forward Secrecy}

It is essential for security protocols that the information transmitted in a session is not compromised, as well as traced or used by an adversary to create vulnerabilities in the current, previous, or future authentication session between the RFID tag and RFID Server $S$. In the proposed protocol, even if the identity $I D_{T}$ or alias identity are lost, it does not affect previous or next sessions. It is ensured through the use of encrypted $A I D_{T i}$, which is updated in every new session. In this way, the proposed protocol for the RFID System guarantees backward and forward secrecy.

\subsubsection{Scalability}

In the proposed protocol for the RFID System, the RFID Server $S$ does not perform an exhaustive process to authenticate any RFID-tag. Instead, the RFID-Server $S$ processes $A I D_{T i}$ to validate the RFID tag and responds quickly to the RFID tag. This makes the proposed protocol more scalable.

\subsubsection{Collision Attack}

If RFID-tags share the same credentials for authentication to access the RFID Server, the protocol may be left vulnerable to a collision attack. In the proposed protocol, every RFID tag uses different 
parameters, i.e., $\left\{N_{y}, R_{i}, V_{2}, M_{A_{1}}\right\}$, for authentication that makes it impossible for collision attack to take place.

\subsubsection{DoS Attack}

The protocol is not based on any random key that is responsible for authentication or verification of the RFID tag; rather, it is based on $A I D_{T i}$ that is well encrypted and updated for every transaction. Therefore, the proposed scheme resists any DoS attack.

\subsubsection{Replay Attacks}

In a replay attack, the attacker may delay or repeat the transmitted information for authentication with the Server $S$. The proposed protocol for RFID systems has three participants: tag, Reader, and Server. For authentication, four messages are exchanged, i.e, $\left\{M_{1}, M_{2}, M_{3}, M_{4}\right\}$, using a public channel. Having access to the messages, an adversary $A$ may attempt to launch a replay attack. However, this attempt will fail as every message is sent with a fresh time-stamp $T$. In case the time-stamp is invalid, the adversary $A$ request will be rejected each time. Furthermore, if an adversary $A$ cannot compute other parameters of the message, the adversary still cannot launch the attack as all message parameters are updated for every new session by the participants of RFID System. Therefore, the proposed protocol for RFID systems is resistant to replay attack.

\subsubsection{Location Tracking Attack}

As the real identity of the RFID tag is not sent directly in the message for authentication between the RFID-tag and Server S, it has been sent in an encrypted form that only the Server can decrypt using its secret key. Moreover, the messages exchanged among the participants are constantly updated in every new session that provides unpredictability. Hence, an adversary cannot find the location and any attempt of finding the location will ultimately fail.

\subsubsection{Impersonation Attacks (Forgery Attacks)}

An adversary $A$ may intercept the messages of the previous legitimate RFID tag and modify that for authentication with the RFID Server $S$. In this case, the adversary $A$ needs to make a valid message request that includes different parameters, like $N_{y}, R_{i}, V_{2}, M_{A_{1}}, A I D_{T i}$. To do so, the adversary $A$ must compute $A I D_{T i}$ that is well encrypted and impossible to be computed or forged. Moreover, the adversary $A$ also needs different other parameters and timestamps to put a valid request for authentication as a legitimate RFID tag. It is impossible for the adversary $A$ without knowing the actual parameters of the Message used for authentication, hence leaving the adversary $A$ unable to prove its legitimacy as an RFID tag to the RFID Server $S$. Reluctantly, the proposed protocol for RFID System resists any forgery attack.

\subsubsection{Stolen-Verifier Attacks}

The proposed protocol resists stolen-verifier-attack. All the verification and validation keys are stored encrypted in the RFID Database Server $S$. If the data and keys are stolen from the RFID Database Server $S$, still the adversary $A$ cannot decrypt and extract them. Also, the adversary $A$ cannot alter or modify the original data saved in the RFID Database Server $S$. Hence, the proposed protocol resists any stolen-verifier attack.

\section{Comparative Analysis}

This section presents a comprehensive comparative analysis of the proposed protocol with the existing protocols $[3,21,23,30,31]$, as these schemes are based on lightweight symmetric key primitives. Hence, they are eligible for a fair comparison with the proposed scheme. Firstly, the proposed protocol is compared with the existing protocols in terms of security requirements. Secondly, a comparison of 
the proposed protocol with existing protocols based on computation cost (running time or execution time) is given, and thirdly, a comparison based on communication cost is presented. Furthermore, the proposed protocol is analyzed for storage complexity. Please note that we have selected the schemes based on lightweight symmetric key primitives and also that have been published recently. Each of these comparisons has been elaborated in the following subsections, one-by-one.

\subsection{Security Requirements}

Security requirements are the features expected from an authentication protocol. Every authentication protocol must be able to ensure these features or requirements. By these requirements, the proposed protocol is compared with the existing protocols. Following is the list of features/requirements considered for comparative analysis.

- SR1: Mutual authentication.

- SR2: Tag untraceability.

- SR3: Tag anonymity.

- SR4: Backward/Forward secrecy.

- SR5: Scalability.

- SR6: Collision attacks.
- SR7: DoS attacks.

- SR8: Replay attacks.

- SR9: Location tracking attack.

- SR10: Forgery attack.

- SR11: Stolen-verifier attacks.

Table 4 shows the security requirements comparison of the proposed protocol with existing symmetric key-based protocols $[3,21,23,30,31]$.

Table 4. Security requirements table.

\begin{tabular}{lcccccc}
\hline Requirements & Yang et al. [23] & Tan et al. [30] & Cai et al. [21] & Cho et al. [31] & Gope et al. [3] & Proposed Scheme \\
\hline SR1 & $\times$ & $\times$ & $\checkmark$ & $\checkmark$ & $\checkmark$ & $\checkmark$ \\
SR2 & $\times$ & $\times$ & $\times$ & $\checkmark$ & $\checkmark$ & $\checkmark$ \\
SR3 & $\times$ & $\times$ & $\checkmark$ & $\times$ & $\checkmark$ & $\checkmark$ \\
SR4 & $\times$ & $\checkmark$ & $\times$ & $\checkmark$ & $\checkmark$ \\
SR5 & $\times$ & $\times$ & $\times$ & $\times$ & $\checkmark$ \\
SR6 & $\times$ & $\times$ & $\times$ & $\checkmark$ & $\checkmark$ \\
SR7 & $\checkmark$ & $\checkmark$ & $\checkmark$ & $\checkmark$ & $\checkmark$ \\
SR8 & $\checkmark$ & $\checkmark$ & $\checkmark$ & $\checkmark$ & $\checkmark$ \\
SR9 & $\checkmark$ & $\checkmark$ & $\checkmark$ & $\times$ & $\checkmark$ \\
SR10 & $\checkmark$ & $\checkmark$ & $\checkmark$ & & \\
SR11 & & $\checkmark:$ Yes provides, $\times$ : Does not provide. &
\end{tabular}

The insecurities of the existing schemes $[3,21,23,30,31]$ are well defined in Section 1 and are replicated in Table 4 . The security requirements in Table 4 show that only the proposed protocol provides all security features.

\subsection{Computation Cost Analysis}

This section describes the computation cost analysis of the proposed protocol with existing related protocols $[3,21,23,30,31]$. For analysis purposes, the following notations are introduced:

- CC: Computation cost;

- $T_{h}$ : CC of single hash function;

- $T_{s e}$ : CC of symmetric encryption/decryption. 
Table 5. Comparison of computation cost and running time.

\begin{tabular}{lllllll}
\hline Computation Cost & Yang et al. [23] & Tan et al. [30] & Cai et al. [21] & Cho et al. [31] & Gope and Hwang [3] & Proposed Scheme \\
\hline$C C_{\text {tag }}$ & $2 T_{h}$ & $2 T_{h}$ & $4 T_{h}$ & $3 T_{h}$ & $5 T_{h}$ & $2 T_{h}$ \\
$C C_{R_{i}}$ & $3 T_{h}$ & $2 T_{h}$ & $2 T_{h}$ & $2 T_{h}$ & $2 T_{h}$ & $2 T_{h}$ \\
$C C_{S}$ & $5 T_{h}$ & $3 T_{h}$ & $6 T_{h}$ & $5 T_{h}$ & $7 T_{h}$ & $4 T_{h}+2 T_{s e}$ \\
$C C_{\text {Total }}$ & $10 T_{h}$ & $7 T_{h}$ & $12 T_{h}$ & $10 T_{h}$ & $14 T_{h}$ & $8 T_{h}+2 T_{s e}$ \\
$C C_{\text {Time }}$ & $0.023 \mathrm{~ms}$ & $0.0161 \mathrm{~ms}$ & $0.0276 \mathrm{~ms}$ & $0.023 \mathrm{~ms}$ & $0.0322 \mathrm{~ms}$ & $0.0276 \mathrm{~ms}$ \\
\hline
\end{tabular}

Table 5 shows the computation cost analysis. The protocol presented in [23] incurs $2 T_{h}, 3 T_{h}$, and $5 T_{h}$, for each tag, Reader and Server, respectively, making its total computation cost $10 T_{h}$. Similarly, the computation cost of protocol presented in [30] is $2 T_{h}, 2 T_{h}$, and $3 T_{h}$, respectively, for each participant, totaling it to $7 T_{h}$. The protocol presented in [21] requires $4 T_{h}, 2 T_{h}$, and $6 T_{h}$ for each tag, Reader, and Server, respectively, totaling it to $12 T_{h}$. The computation cost of the protocol of Gope and Hwang [3] is $5 T_{h}, 2 T_{h}$, and $7 T_{h}$, respectively, for each participant totaling it to $14 T_{h}$. In comparison, the tag in proposed protocol uses $2 T_{h}$, the Reader uses $2 T_{h}$, and the Server requires $4 T_{h}+2 T_{s e}$, so in total the computation cost of the proposed protocol is equal to $8 T_{h}+2 T_{s e}$. Considering the experiment of Kilinc and Yanik [47], the computation time of $T_{h}$ is $0.0023 \mathrm{~ms}$, whereas the computation time to calculate $T_{s e}$ is $0.0046 \mathrm{~ms}$. The experiment was performed on a Ubuntu system with an Intel dual-core Pentium processor with specifications, including $2.20 \mathrm{GHz}, 2048 \mathrm{MB}$ processor and Ram, respectively. The total computation time of the proposed protocol is $0.0276 \mathrm{~ms}$, whereas the total cost of the protocol presented in [23] is $0.0230 \mathrm{~ms}$, the cost of protocol in [30] is approximately $0.0161 \mathrm{~ms}$, the cost of the proposal in [3] is $0.0322 \mathrm{~ms}$, and the proposal in [21] takes a total of $0.0276 \mathrm{~ms}$. Although the proposed protocols incur a slightly higher computation cost as compared with [23,30], it provides less computation cost when compared with the baseline [3] and provides the same computation cost as compared to the protocol presented in [21]. Moreover, the proposed protocol is the only protocol that provides resistance against all known attacks. The results presented in Table 5 are visualized in Figure 7.

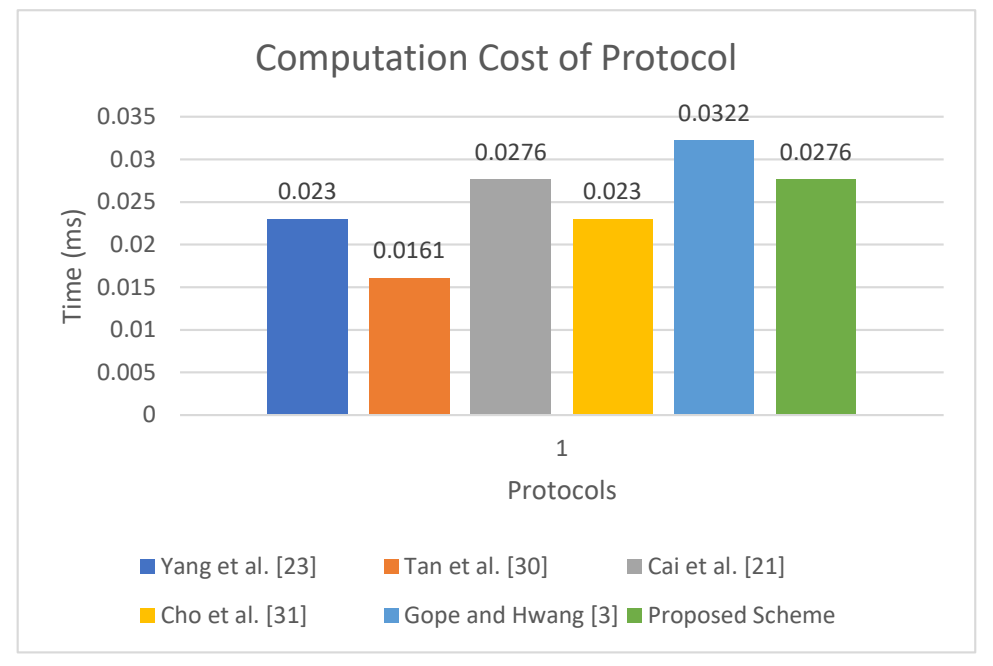

Figure 7. Running Time of Proposed Scheme.

\subsection{Communication and Storage Cost Analysis}

Communication cost is presented in terms of the total number of messages exchanged and total number of bits exchanged during one transaction of the protocol. In the proposed protocol, $\mathrm{tag}_{i}$ transmits four parameters in $M_{1}$ to $R_{i}$ carrying 416 bits and receives 384 bits from $R_{i}$. Similarly, $R_{i}$ transmits 736 bits and receives 416 bits from $S$, while $S$ transmits 416 bits and receives 736 bits. The communication cost comparison of the proposed protocol with other existing protocols is presented in Table 6 . The storage cost is represented by length Value $L$, the proposed protocol uses $S H A-1$ hash function to implement $h($.$) ; for simplicity, each of the length values is considered as 160-bit long.$ 
In the proposed scheme, each tag stores $I D_{T_{i}}, K_{t s}, A I D$ parameters. Therefore, the cost of storage in the tag is $3 L$, whereas on the Server side, $I D_{T_{i}}, K_{t s}, A I D_{\text {new }}, A I D_{\text {old }}$ are being stored; hence, the storage cost on the Server side is $4 L$ per tag.

Table 6. Communication Cost of Proposed and other Protocols.

\begin{tabular}{lccccc}
\hline Schemes & tag & Reader & Server & Total Bits & Messages \\
\hline Yang et al. [23] & 256 & 512 & 640 & 1408 & 5 \\
Tan et al. [30] & 896 & 768 & 768 & 2432 & 4 \\
Cai et al. [21] & 256 & 544 & 256 & 1056 & 5 \\
Cho et al. [31] & 512 & 512 & 256 & 1280 & 5 \\
Gope and Hwang [3] & 416 & 1180 & 288 & 1888 & 4 \\
Proposed Protocol & 416 & 736 & 416 & 1568 & 4 \\
\hline
\end{tabular}

The proposed protocol incurs less communication cost as compared with the protocols of $[3,30]$, whereas it has more communication cost when compared with others [21,23,31]. However, only the proposed protocol provides required security. The results presented in Table 6 are visualized in Figure 8.

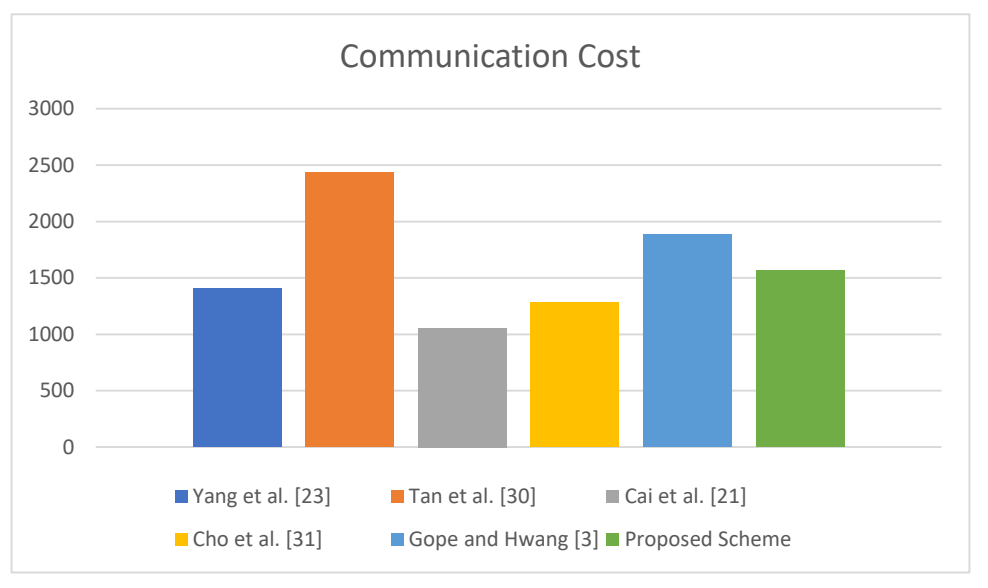

Figure 8. Communication Cost.

Table 6 indicates that the proposed protocol is more efficient than the baseline protocol in terms of communication cost. Specifically, the proposed protocol not only overcomes the security flaws of the baseline protocol but also achieves $16.94 \%$ efficiency in terms of the number of bits exchanged during one transaction of the protocol.

\section{Conclusions}

In this article, cryptanalysis of a recent authentication protocol by Gope and Hwang has been presented, and it has been proved that their protocol has some weaknesses against collision, stolen verifier, and DoS attacks. An improved scheme using only lightweight primitives is proposed to resist all known attacks. The security of the proposed scheme has been thoroughly analyzed informally, as well as formally, using BAN logic. Moreover, the scheme is simulated in automated applied $\pi$ calculus-based tool ProVerif. The simulation also backs the formal and informal security analysis. Although the proposed scheme incurs some extra computation and communication cost as compared with some existing related protocols, only the proposed protocol resists all known attacks and is more suitable for practical IoT-based scenarios. 
Author Contributions: For research articles with several authors, conceptualization, K.M., S.A.C. and A.G.; methodology, K.M. and S.A.C.; software, K.M.; validation, A.G., S.S., A.M., and S.A.K.G.; formal analysis, S.A.C. and A.G.; investigation, K.M.; resources, S.S. and A.M.; data curation, K.M. and A.G.; writing-original draft preparation, K.M. and A.G.; writing-review and editing, S.A.C. and A.G.; visualization, A.G.; supervision, A.G. and S.A.C.; project administration, A.G. and S.A.C.; funding acquisition, S.S. and A.M.

Funding: This research received no external funding.

Conflicts of Interest: The authors declare that they have no conflict of interest.

\section{References}

1. Rouse, M. Internet of Things (IoT). Available online: https://internetofthingsagenda.techtarget.com/ definition/Internet-of-Things-IoT (accessed on 3 September 2019).

2. Gope, P.; Hwang, T. BSN-Care: A secure IoT-based modern healthcare system using body sensor network. IEEE Sens. J. 2016, 16, 1368-1376. [CrossRef]

3. Gope, P.; Hwang, T. A realistic lightweight authentication protocol preserving strong anonymity for securing RFID system. Comput. Secur. 2015, 55, 271-280. [CrossRef]

4. Peris-Lopez, P.; Hernandez-Castro, J.C.; Estevez-Tapiador, J.M.; Ribagorda, A. Lightweight cryptography for low-cost RFID tags. In Security in RFID and Sensor Networks; CRC Press: London, UK, 2016; pp. 121-150.

5. Gope, P.; Amin, R.; Islam, S.H.; Kumar, N.; Bhalla, V.K. Lightweight and privacy-preserving RFID authentication scheme for distributed IoT infrastructure with secure localization services for smart city environment. Future Gener. Comput. Syst. 2018, 83, 629-637. [CrossRef]

6. Kitsos, P. Security in RFID and Sensor Networks; CRC Press: New York, NY, USA, 2016.

7. Hsu, C.H.; Wang, S.; Zhang, D.; Chu, H.C.; Lu, N. Efficient identity authentication and encryption technique for high throughput RFID system. Secur. Commun. Netw. 2016, 9, 2581-2591. [CrossRef]

8. Simon, P.M.G.; Riggert, E.F.; Trivelpiece, S.E. System and Method for Reading RFID Tags Across a Portal. U.S. Patent 9,519,811, 13 December 2016.

9. Wu, F.; Xu, L.; Kumari, S.; Li, X.; Das, A.K.; Shen, J. A lightweight and anonymous RFID tag authentication protocol with cloud assistance for e-healthcare applications. J. Ambient Intell. Humanized Comput. 2018, 9, 919-930. [CrossRef]

10. Sidorov, M.; Ong, M.T.; Vikneswaran, R.; Nakamura, J.; Ohmura, R.; Khor, J.H. Ultralightweight Mutual Authentication RFID Protocol for Blockchain Enabled Supply Chains. IEEE Access 2019, 7, 7273-7285. [CrossRef]

11. Noman, A.T.; Hossain, S.; Islam, S.; Islam, M.E.; Ahmed, N.; Chowdhury, M.M. Design and Implementation of Microcontroller Based Anti-Theft Vehicle Security System using GPS, GSM and RFID. In Proceedings of the 2018 4th International Conference on Electrical Engineering and Information \& Communication Technology (iCEEiCT), Dhaka, Bangladesh, 13-15 September 2018; pp. 97-101.

12. Liao, Y.P.; Hsiao, C.M. A secure ECC-based RFID authentication scheme integrated with ID-verifier transfer protocol. Ad Hoc Netw. 2014, 18, 133-146. [CrossRef]

13. Kim, H. RFID mutual authentication protocol based on synchronized secret. Int. J. Secur. Its Appl. 2013, 7, 37-50.

14. Cha, J.R.; Kim, J.H. Novel anti-collision algorithms for fast object identification (RFID) system. In Proceedings of the 11th International Conference on Parallel and Distributed Systems, Washington, DC, USA, 20-22 July 2005; Volume 2; pp. 63-67.

15. El Beqqal, M.; Azizi, M. Classification of major security attacks against RFID systems. In Proceedings of the International Conference on Wireless Technologies, Embedded and Intelligent Systems (WITS), Fez, Morocco, 19-20 April 2017; pp. 1-6.

16. Tewari, A.; Gupta, B. Cryptanalysis of a novel ultra-lightweight mutual authentication protocol for IoT devices using RFID tags. J. Supercomput. 2017, 73, 1085-1102. [CrossRef]

17. Ayaz, U.; Haq, T.A.; Taimour, S.; Mansoor, K.; Mahmood, S. An Enhanced Biometric Based RFID Authentication Scheme Defending Against Illegitimate Access. In Proceedings of the 14th International Conference on Emerging Technologies (ICET), Islamabad, Pakistan, 21-22 November 2018; pp. 1-6.

18. Zhao, Z. A secure RFID authentication protocol for healthcare environments using elliptic curve cryptosystem. J. Med. Syst. 2014, 38, 46. [CrossRef] 
19. Farash, M.S.; Nawaz, O.; Mahmood, K.; Chaudhry, S.A.; Khan, M.K. A provably secure RFID authentication protocol based on elliptic curve for healthcare environments. J. Med. Syst. 2016, 40, 165. [CrossRef] [PubMed]

20. Burmester, M.; De Medeiros, B.; Motta, R. Robust, anonymous RFID authentication with constant key-lookup. In Proceedings of the 2008 ACM symposium on Information, computer and communications security, Tokyo, Japan, 18-19 March 2008; pp. 283-291.

21. Cai, S.; Li, Y.; Li, T.; Deng, R.H. Attacks and improvements to an RIFD mutual authentication protocol and its extensions. In Proceedings of the second ACM conference on Wireless network security, Zurich, Switzerland, 16-19 March 2009; pp. 51-58.

22. Gaubatz, G.; Kaps, J.P.; Ozturk, E.; Sunar, B. State of the art in ultra-low power public key cryptography for wireless sensor networks. In Proceedings of the Third IEEE International Conference on Pervasive Computing and Communications Workshops, PerCom Workshops, Kauai Island, HI, USA, 8-12 March 2005; pp. 146-150.

23. Yang, J.; Park, J.; Lee, H.; Ren, K.; Kim, K. Mutual authentication protocol. In Proceedings of the Workshop on RFID and lightweight crypto, Graz, Austria, 14-15 July 2005.

24. Kang, S.Y.; Lee, I.Y. A Study on low-cost RFID system management with mutual authentication scheme in ubiquitous. In Proceedings of the Asia-Pacific Network Operations and Management Symposium, Sapporo, Japan, 10-12 October 2007; pp. 492-502.

25. Lee, L.S.; Fiedler, K.D.; Smith, J.S. Radio frequency identification (RFID) implementation in the service sector: A customer-facing diffusion model. Int. J. Prod. Econ. 2008, 112, 587-600. [CrossRef]

26. Qingling, C.; Yiju, Z.; Yonghua, W. A minimalist mutual authentication protocol for RFID system \& BAN logic analysis. In Proceedings of the International Colloquium on Computing, Communication, Control, and Management, CCCM, Guangzhou, China, 3-4 August 2008, Volume 2; pp. 449-453.

27. Zhou, S.; Zhang, Z.; Luo, Z.; Wong, E.C. A lightweight anti-desynchronization RFID authentication protocol. Inf. Syst. Front. 2010, 12, 521-528. [CrossRef]

28. Piramuthu, S. RFID mutual authentication protocols. Decis. Support Syst. 2011, 50, 387-393. [CrossRef]

29. Safkhani, M.; Peris-Lopez, P.; Hernandez-Castro, J.C.; Bagheri, N. Cryptanalysis of the Cho et al. protocol: A hash-based RFID tag mutual authentication protocol. J. Comput. Appl. Math. 2014, 259, 571-577. [CrossRef]

30. Tan, C.C.; Sheng, B.; Li, Q. Secure and serverless RFID authentication and search protocols. IEEE Trans. Wirel. Commun. 2008, 7, 1400-1407. [CrossRef]

31. Cho, J.S.; Jeong, Y.S.; Park, S.O. Consideration on the brute-force attack cost and retrieval cost: A hash-based radio-frequency identification (RFID) tag mutual authentication protocol. Comput. Math. Appl. 2015, 69, 58-65. [CrossRef]

32. Naeem, M.; Chaudhry, S.A.; Mahmood, K.; Karuppiah, M.; Kumari, S. A scalable and secure RFID mutual authentication protocol using ECC for Internet of Things. Int. J. Commun. Syst. 2019. [CrossRef]

33. Zhang, Z.; Qi, Q. An efficient RFID authentication protocol to enhance patient medication safety using elliptic curve cryptography. J. Med. Syst. 2014, 38, 47. [CrossRef]

34. Chaudhry, S.A.; Naqvi, H.; Farash, M.S.; Shon, T.; Sher, M. An improved and robust biometrics-based three factor authentication scheme for multiserver environments. J. Supercomput. 2018, 74, 3504-3520. [CrossRef]

35. Asgari, H.; Haines, S.; Rysavy, O. Identification of Threats and Security Risk Assessments for Recursive Internet Architecture. IEEE Syst. J. 2018, 12, 2437-2448. [CrossRef]

36. Abbasinezhad-Mood, D.; Nikooghadam, M. An Anonymous ECC-Based Self-Certified Key Distribution Scheme for the Smart Grid. IEEE Trans. Ind. Electron. 2018, 65, 7996-8004. [CrossRef]

37. Tan, H.; Ma, M.; Labiod, H.; Boudguiga, A.; Zhang, J.; Chong, P.H.J. A Secure and Authenticated Key Management Protocol (SA-KMP) for Vehicular Networks. IEEE Trans. Veh. Technol. 2016, 65, 9570-9584. [CrossRef]

38. Chaudhry, S.A.; Kim, I.L.; Rho, S.; Farash, M.S.; Shon, T. An improved anonymous authentication scheme for distributed mobile cloud computing services. Cluster Comput. 2019, 22, 1595-1609. [CrossRef]

39. Roy, S.; Chatterjee, S.; Das, A.K.; Chattopadhyay, S.; Kumari, S.; Jo, M. Chaotic Map-Based Anonymous User Authentication Scheme With User Biometrics and Fuzzy Extractor for Crowdsourcing Internet of Things. IEEE Internet Things J. 2018, 5, 2884-2895. [CrossRef] 
40. Jiang, Q.; Zeadally, S.; Ma, J.; He, D. Lightweight three-factor authentication and key agreement protocol for internet-integrated wireless sensor networks. IEEE Access 2017, 5, 3376-3392. [CrossRef]

41. Mahmood, K.; Naqvi, H.; Alzahrani, B.A.; Mehmood, Z.; Irshad, A.; Chaudhry, S.A. An ameliorated two-factor anonymous key exchange authentication protocol for mobile client-server environment. Int. J. Commun. Syst. 2018, 31, e3814. [CrossRef]

42. Xu, Z.; Xu, C.; Chen, H.; Yang, F. A lightweight anonymous mutual authentication and key agreement scheme for WBAN. Concurr. Comput. Pract. Exp. 2019, 31, e5295. [CrossRef]

43. Xie, Q.; Hwang, L. Security enhancement of an anonymous roaming authentication scheme with two-factor security in smart city. Neurocomputing 2019, 347, 131-138. [CrossRef]

44. Kyntaja, T. A logic of authentication by Burrows, Abadi and Needham; Science Helsinki University of Technology: Tehran, Iran. Available online: http:/ / www.tml.tkk.fi/Opinnot/Tik-110.501/1995/ban.html (accessed on 13 July 2019).

45. Blanchet, B. Modeling and verifying security protocols with the applied pi calculus and ProVerif. Found. Trends Privacy Secur. 2016, 1, 1-135. [CrossRef]

46. Lumini, A.; Nanni, L. An improved biohashing for human authentication. Pattern Recognit. 2007, 40, 1057-1065. [CrossRef]

47. Kilinc, H.H.; Yanik, T. A survey of SIP authentication and key agreement schemes. IEEE Commun. Surv. Tutor. 2014, 16, 1005-1023. [CrossRef]

(C) 2019 by the authors. Licensee MDPI, Basel, Switzerland. This article is an open access article distributed under the terms and conditions of the Creative Commons Attribution (CC BY) license (http:/ / creativecommons.org/licenses/by/4.0/). 